ACTA UNIVERSITATIS LODZIENSIS

Folia Litteraria Romanica 16, 2021

https://doi.org/10.18778/1505-9065.16.01

Alicja Kacprzak

Université de Łódź

(iD) https://orcid.org/0000-0002-3113-8534

alicja.kacprzak@uni.lodz.pl

Agnieszka Woch

Université de Łódź

(iD) https://orcid.org/0000-0003-0559-9166

agnieszka.woch@uni.lodz.pl

\title{
Avant-propos
}

\section{Des variétés de langue à la linguistique sociale urbaine : en hommage à Jean-Pierre Goudaillier}

Ce volume est un recueil de contributions dédiées à Jean-Pierre Goudaillier par ses collègues et disciples à l'occasion de son $70^{\circ}$ anniversaire. Réunis autour de la question, si chère au Professeur, des variations de la langue, ils souhaitent rendre hommage à celui dont l'apport scientifique est pour ce domaine essentiel. En effet, la recherche de Jean-Pierre Goudaillier consacrée aux usages périphériques non normés des langues, et plus particulièrement du français contemporain des cités (FCC), s'inscrit dans le cadre d'une argotologie générale que le Professeur situe entre la linguistique et la sociologie. C'est par cette perspective novatrice que Jean-Pierre Goudaillier propose, dans ses nombreux travaux, les fondements d'une linguistique sociale urbaine qui chercherait l'explication des faits linguistiques dans des facteurs relevant de la condition sociale des locuteurs.

Peu le savent, mais la recherche de Jean-Pierre Goudaillier, né le 14 décembre 1949 à Alger, n'a pas toujours porté sur les variantes sociolectales du français : ses premières études ont été consacrées à la phonologie et la phonétique du luxembourgeois. Élève d'André Martinet, lors de ses études à l'Université Paris 5 à partir de 1968, il publie en 1973 au Grand-Duché du Luxembourg l'ouvrage intitulé Les relations spatiales en luxembourgeois. À partir de corpus de diverses variétés de luxembourgeois, de français et de québécois, entre autres langues, il établit les fondements d'une Phonologie Fonctionnelle Expérimentale (P.F.E.), 
qui assoit les liens entre phonologie fonctionnelle et phonétique expérimentale. Il est nommé Maître de Conférences de linguistique et phonétique en 1978, puis Professeur de linguistique générale en 1987 à l'Université René Descartes de Paris.

C'est au cours des années 1980 que Jean-Pierre Goudaillier opère une conversion thématique « argotologique » et crée à la Sorbonne en 1986 avec Denise François-Geiger et Marc Sourdot le Centre de Recherches Argotologiques CARGO, tout d'abord rattaché à Paris 13 au Laboratoire de Linguistique Informatique (dirigé par Gaston Gross), avant de devenir sous sa responsabilité la sous-équipe PAVI (Productions Argotiques et Variations Interculturelles) de l’Équipe d'Accueil (E.A.) 1643 (3790) DYNALANG (Théorie linguistique et analyse de la dynamique des langages humains), E.A. qu'il dirige jusqu'en 2009. Les travaux de recherche de Jean-Pierre Goudaillier se situent dès lors dans les domaines lexicographique et argotologique. Il publie en 1997 à Paris l'ouvrage intitulé Comment tu tchatches! Dictionnaire du Français Contemporain des Cités $(F C C)$ chez Maisonneuve \& Larose. " Ce livre impeccable, qui prend forcément et sympathiquement le parti de ce dont il traite $»^{1}$ est ensuite réédité en 1998 et en 2001. La nouvelle édition de ce dictionnaire de référence, très attendue, voit le jour, revue et augmentée, en novembre 2019, chez Maisonneuve \& Larose / Hémisphères.

Enseignant et maître par excellence, Jean-Pierre Goudaillier a été aussi le fondateur d'un séminaire d'argotologie dans son université, qui a rassemblé pendant des années des dizaines d'étudiants français et étrangers attirés par sa thématique originale et passionnante. Plusieurs doctorats, dont certains issus de ces séminaires, dirigés ou co-dirigés par Jean-Pierre Goudaillier avec des partenaires d'universités étrangères, ont porté sur différents aspects de la variation sociale en français et dans d'autres langues. L'éventail de thématiques abordées est impressionnant, cela va du parler des marins de l'École navale aux argotismes d'origine carcérale en français, en passant par la langue du slam et du rap, ou par l'argot des étudiants hongrois ou tchèques et les gros mots dans l'espagnol du Mexique. C'est grâce à ce « vivier » argotologique que par la suite plusieurs chaires étrangères de romanistique ont incorporés dans leurs études des cours et séminaires centrés sur le français non standard, en enrichissant de cette façon leur offre didactique et scientifique (c'est le cas entre autres des universités de Brno, de Budapest, de Łódź).

C'est à la même époque aussi que Jean-Pierre Goudaillier a lancé le projet d'organiser chaque année un colloque d'argotologie, en continuation de celui de Besançon de 1989. Ainsi, à partir de 2005 où les argotologues se sont réunis à Budapest, les rencontres se sont succédé, ayant lieu : à Łódź en 2008, à Brno en 2010, à Leipzig en 2011, à Innsbruck en 2012, à Łódź en 2013, à Budapest

\footnotetext{
${ }^{1}$ Pierre Mayol (1998), « Jean-Pierre Goudaillier - Comment tu tchatches ! Dictionnaire du français contemporain des cités » [note bibliographique] in Agora débats/jeunesses nº 12, p. 152.
} 
en 2014, à Alicante en 2015, à Ljubljana en 2016, à Leipzig en 2017, à Łódź en 2018 et à Paris en 2019. Comme résultat de ces rencontres, plusieurs volumes présentant différentes vues sur la problématique des variétés de langues, co-rédigés par Jean-Pierre Goudaillier avec les collègues des centres énumérés ont été publiés. L'ensemble de ces publications constitue aujourd'hui une sorte de compendium unique et exceptionnel de cette branche de la linguistique.

Les activités scientifique et didactique du Professeur ont été, tout au long de ses années de travail, accompagnées par ses nombreuses responsabilités au sein de l'université. De 1990 à 1999, il a exercé la fonction de directeur de l'U.F.R. de Linguistique Générale et Appliquée, puis de 1999 à 2007, celle de doyen de la Faculté des Sciences Humaines et Sociales - Sorbonne de l'Université Paris Descartes. Depuis septembre 2018, Jean-Pierre Goudaillier est professeur émérite de l'Université de Paris (Université Paris Descartes), mais ne renonce ni à ses tâches de directeur de thèse, ni à sa plume de chercheur, et il n'a rien perdu de son ardeur d'organisateur d'échanges scientifiques internationaux.

\section{Ad multos annos, Cher Professeur!}

Le présent volume, dédié à Jean-Pierre Goudaillier, s'interroge sur les moyens d'exprimer l'amour dans différentes variations de la langue et rassemble des études relevant de plusieurs domaines couverts par les sciences du langage et les lettres. Le recueil compte 23 articles répartis en fonction de six thématiques ainsi que les comptes rendus de deux ouvrages parus en 2019.

Les trois premiers textes proposent une analyse linguistique du phénomène étudié en se centrant sur les conceptualisations et formulations des termes « amour » et « aimer». Anna Bobińska se penche sur les formes d'adresse affectives dans le récit bédéistique en mettant l'accent sur celles qui font appel aux registres non-standard de langue. L'auteure décrit les traits énonciatifs et pragmatiques caractéristiques d'un discours basé sur la composante affective et examine le contenu sémantique des formes d'adresse, leurs valeurs fonctionnelles et leur dimension expressive. Malgorzata Izert et Ewa Pilecka se livrent à une recherche sur un corpus visant à rendre compte du fonctionnement des intensifieurs en français familier, populaire et argotique. Les auteures examinent les termes permettant d'exprimer une intensité forte et faible qu'elles combinent avec les lexies amour, amoureux, aimer. Magdalena Lipińska s'intéresse à la conceptualisation de la notion d'amour dans les citations et proverbes français et polonais. L'auteure isole six Modèles Cognitifs Idéalisés qu'elle constate être communs aux deux langues en soulignant toutefois que les Français comprennent plus souvent l'amour dans les catégories des universaux culturels par rapport aux Polonais. 
Quatre articles regardent les aspects contrastifs et interculturels de l'amour. La contribution d'Anna Bochnakowa vise à expliquer le fonctionnement des mots amour/miłość et aimer/kochać pour mettre en évidence leur sens et leur emploi en français et en polonais. Cette analyse permet de constater une grande richesse synonymique du verbe français aimer et le fait que les champs sémantiques des mots amour et aimer, appartenant cependant aux universaux du langage, diffèrent dans les deux langues. L'étude comparative de Joanna Cholewa est consacrée à la locution française tomber amoureux et sur le verbe polonais préfixé zakochać się. L'auteure en propose une fine analyse qui démontre que tomber amoureux exprime un changement d'état et souligne le début de l'action, tandis que zakochać się met en évidence l'intensité du sentiment auquel se réfère la base verbale. La contribution de Verónica Rodriguez Ferreiro est une réflexion sur l'image de la femme dans le cadre d'une parémiologie comparée et une contribution au dictionnaire phraséologique français-espagnol-galicien. Son champ de travail est restreint aux proverbes associés à l'amour et plus particulièrement à la figure féminine. L'auteure constate que les équivalences sont rarement pleines entre les proverbes français, espagnols et galiciens. Alma Sokolija livre une étude interculturelle sur l'articulation de l'amour et la perception de la sexualité dans les argots du monde. Elle part de l'hypothèse que certaines notions tabous ne subissent pas de changements surtout dans les sociétés majoritairement patriarcales. L'auteure analyse des expressions qui proviennent du français mais aussi de l'arabe, du chinois mandarin et du japonais.

Les contributions qui suivent s'interrogent sur différentes variétés de la langue. Gueorgui Armianov porte un regard critique sur le vocabulaire bulgare argotique et familier lié à l'amour et au sexe. L'auteur accorde une attention particulière à la désargotisation de plusieurs lexèmes et à leur intégration progressive dans le langage familier et dans la pratique langagière courante. La recherche de Máté Kovács se concentre sur l'analyse du champ lexical d'aimer dans l'argot commun des jeunes Hongrois. Il se propose premièrement de vérifier le degré d'utilisation des mots et expressions liés au champ lexical d'aimer présents dans le dictionnaire de référence de Dávid Szabó. Il élabore et examine ensuite un nouveau corpus en réfléchissant sur l'usage actuel des lèxemes. Tatiana Retinskaya se penche sur les appellatifs affectifs régionaux et démontre qu'ils sont un moyen de sauvegarder le parler spécifique local. Son étude du vocabulaire régional champenois et ardennais comprend la description de différents types de transpositions affectueuses utilisées dans l'interlocution et l'analyse des variétés morphosémantiques. L'article de Jean-François Sablayrolles constitue une réflexion sur les verbes et expressions dénommant l'acte sexuel en mettant en évidence la domination du point de vue masculin dont ils témoignent. Rares en français académique, l'auteur constate leur haute fréquence en français non conventionnel et observe que l'argot se renouvelle et se manifeste par une inventivité littéraire importante. 
La quatrième partie du recueil porte sur « l'amour et aimer » dans différents types de discours. Mateusz Bialas appuie son analyse sur un matériau variationnel diastratique relevant du non-standard et constitué par une centaine de biographies érotiques d'acteurs X homo-et bisexuels. Il s'attacheà démontrer comment la langue française contemporaine aborde l'amour physique à l'époque du culte du corps et des nouvelles technologies. Florian Koch et Marie-Anne Berron effectuent une analyse de contenu des forums liés au foot et s'interrogent sur les expressions de l'amour et de dédain exprimés par les supporters. Leur étude démontre que les expressions haineuses qui démarquent les groupes sont déterminantes pour la formation de leur identité collective. Agnieszka Konowska analyse le discours numérique sur le couple présidentiel français afin de déterminer comment les stéréotypes concernant l'amour intergénérationnel servent d'arguments contre la politique d'Emmanuel Macron. Selon l'auteure, le recours aux stéréotypes relève d'une argumentation ad personam et le caractère « anti-doxique » de la relation du président avec sa femme devient un instrument de délégitimation de sa personne et de sa politique. Agnieszka Woch met en avant les stratégies discursives présentes dans le discours médiatique polonais. Sa contribution constitue une réflexion sur l'emploi des termes « patriote », " patriotisme », " patriotique » et s'appuie sur un corpus d'articles publiés dans des journaux numériques. L'auteure constate que dans les discours analysés, le mot " patriote» devient une étiquette, à la fois noble et stigmatisante, en fonction de l'orientation politique de la presse examinée.

Les contributions qui suivent s'inscrivent dans le contexte des recherches scientifiques dédiées à l'onomastique. Stéphane Hardy s'intéresse à l'usage des pseudonymes de prostituées exerçant leur métier à Paris entre le XVIII ${ }^{\mathrm{e}}$ siècle et le début du $\mathrm{XX}^{\mathrm{e}}$ siècle. Elle applique à ce corpus, recueilli dans des rapports de police et dans les ouvrages sociologiques de l'époque, une taxonomie distinguant plusieurs types de procédés, tant morphosyntaxiques que sémantiques, de formation des pseudonymes. Alicja Kacprzak et Andrzej Napieralski abordent la question des appellations non officielles des équipes de football françaises, espagnoles et polonaises. Cette analyse des formes, du contenu et des rôles des appellations officieuses permet de proposer une nette distinction entre les surnoms et les sobriquets et de souligner qu'ils constituent deux catégories de chrématonymes bien distinctes. Radka Mudrochová et Nyeberth Emanuel Pereira dos Santos étudient les surnoms affectifs en France et au Québec à partir d'un corpus de mots recueillis et analysés avec des outils de la linguistique de corpus. Les auteurs proposent une classification des surnoms affectifs, se penchent sur leur formation, leur fréquence et leur productivité ce qui permet de mettre en évidence les points communs et les différences dans l'emploi des formes affectives dans les deux pays fracophones.

La dernière partie du volume porte sur l'amour et aimer dans les œuvres littéraires et dans la chanson. Mieczyslaw Gajos examine les contextes d'utilisation du nom amour et du verbe aimer dans les paroles des chansons d'Édith Piaf afin de 
relever l'ensemble du lexique lié à ce champ lexical et d'analyser leur importance dans l'œuvre de la chanteuse. Cette analyse linguistique est complétée par une réflexion didactique sur la possibilité d'utiliser les chansons du répertoire de Piaf dans les cours de français comme langue étrangère. Olga Stepanova livre une étude sur le parler des jeunes dans le roman contemporain. L'auteure considère que l'amour est un thème nouveau dans la perception de la banlieue. Sa recherche s'inscrit dans les études de genre et révèle la tension entre le sentiment d'amour éprouvé par les adolescents et l'acte sexuel. L'article de Dávid Szabó constitue une intéressante réflexion sur l'amour physique dans les chansons de Georges Brassens. L'analyse qualitative du vocabulaire lié à la sexualité met en évidence une grande variabilité stylistique de l'auteur, qui aborde souvent le thème sans être explicite, et permet de constater que le recours à l'argot et aux termes grossiers chez Brassens, qui a pourtant une réputation de libertin et d'auteur-interprète de chansons à scandale, ne se caractérise pas par une fréquence particulièrement élevée. Lukasz Szkopiński se penche sur le thème de l'amour dans Les Ombres sanglantes (1820) de J. P. R. Cuisin et analyse quatre histoires de ce recueil en s'intéressant particulièrement aux crimes qui y sont décrits et à la manière violente dont les différentes relations sentimentales, souvent de nature illicite, sont représentées dans l'ouvrage. Il est montré que la vision de l'amour dans Les Ombres sanglantes ne ressemble pas à celle du roman noir de l'époque et qu'elle marque l'avènement du genre frénétique dans la littérature française de cette période. Marina Tikhonova aborde le thème de l'amour dans la poésie française pour les enfants et l'étudie du point de vue de différentes variations de la langue. L'auteure examine le lexique de textes poétiques, y compris le lexique affectif, argotique et familier en arrivant à la conclusion que le ton des poèmes va du lyrisme à l'espièglerie et que l'humour qui y est présent correspond parfaitement au caractère ludique de la poésie pour enfants.

Le volume se clôt sur les deux comptes rendus d'Alicja Kacprzak et de Magdalena Szeflińska-Baran qui proposent une lecture critique de deux ouvrages récemment parus sur la néologie. 